\title{
COMBINED INFLUENCE OF HALL CURRENT AND SORET EFFECT ON CONVECTIVE HEAT AND MASS TRANSFER FLOW PAST A VERTICAL POROUS PLATE IN A ROTATING FLUID AND DISSIPATION WITH CIONSTANT HEAT AND MASS FLUX AND PARTIAL SLIP
}
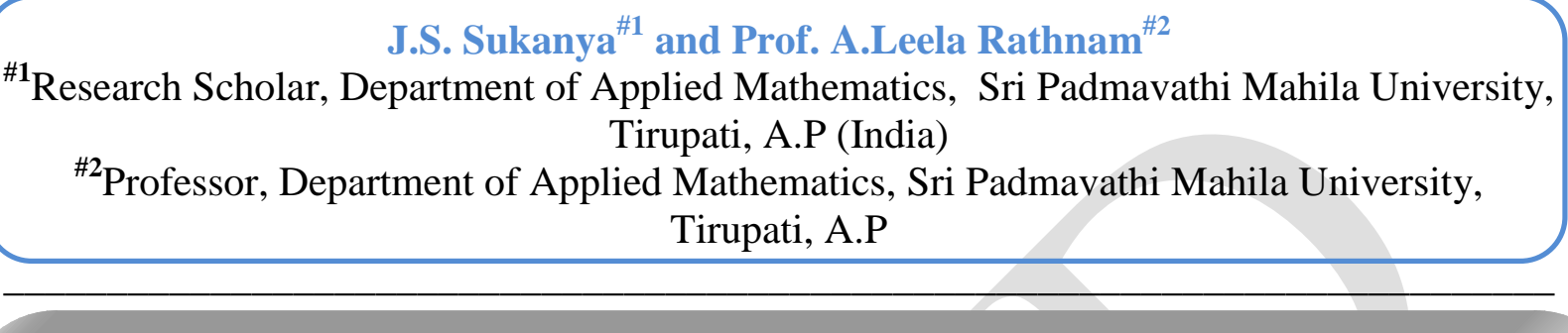

\section{ABSTRACT}

In this paper we analyse the effect of thermo-diffusion on convective heat and mass transfer flow of viscous electrically conducting rotating fluid in a vertical rotating plate in the presence of transverse magnetic field. By employing finite element technique the equations governing the flow, heat and mass transfer have been solved. The velocity and temperature dissipations are analysed for a different parametric values. The shear stress and rate of heat and mass transfer on the boundary are evaluated numerically for different variations.

Key Words: Hall Currents, Soret Effect, Rotating fluid, Constant heat and mass flux, partial slip

\section{INTRODUCTION}

Studies associated with flow through porous medium in a rotating environment have some relevance in a geophysical, geothermal. Many aspects of motion in rotating frame of reference of terrestrial and planetary atmosphere are influenced by the effects of rotation of the medium.

It is well known that in the geothermal region, gases are electrically conduction and that they undergo the influence of magnetic fluid. It is challenging approach to atmospheric science that exerts its influence of rotation to help in understanding the behavior of oceanic circulation and formation of galaxies. The effect of Coriolis force in the atmosphere is exposed to oceanic circulation and the formation of galaxies in taking into account the flow of electron is continuously liberated from the sun what is called "solar wind". The MHD flow in the rotating environment leads to a startup process implying thereby a viscous layer at the boundary is suddenly set into motion and the rate of rotation becomes important in the application of various branches of geophysics, astrophysics and fluid engineering. Several authors [Rao et al[18], Sarojamma and Krishna[19], Krishna et al[14],Seth and ghosh[21],Agarwal and Dhanpal[4], Ghouse[10], El-mistikawy et al.[9], Hazim Ali Attia[11]].

Circar and Mukherjee [7] have analyzed the effect of mass transfer and rotation on flow past a porous plate in a porous medium with variable suction in a slip flow regime. Balasubramanyam [5] and Reddy [15] have investigated convective heat and mass transfer flow in horizontal rotating fluid under different conditions. 
Ibrahim et. al. [12] analyzed the effects of the chemical reaction and radiation absorption on the unsteady MHD free convection flow past a semi-infinite vertical permeable moving plate with heat source and suction. Indudhar et. al. [13] has investigated the effect of chemical reaction and radiation absorption on unsteady MHD double diffusive convective flow of a viscous fluid past a semi-infinite vertical porous plate. Cheena Kesavaiah et.al. [6] have discussed the effects of the chemical reaction and radiation absorption on an unsteady MHD convective heat and mass transfer flow past a semi-infinite vertical permeable moving plate embedded in a porous medium with heat source and suction.

In all these investigations, the effects of Hall currents are not considered. However, in a partially ionized gas, there occurs a Hall current when the strength of the impressed magnetic field is very strong. These Hall effects play a significant role in determining the flow features. Sato [23], Yamanishi [28], Sherman and Sutton [25] have discussed the Hall effects on the steady hydromagnetic flow between two parallel plates. Debnath [8] has studied the effects of Hall currents on unsteady hydromagnetic flow past a porous plate in a rotating fluid system and the structure of the steady and unsteady flow is investigated. Alam et. al.,[2] have studied unsteady free convective heat and mass transfer flow in a rotating system with Hall currents, viscous dissipation and Joule heating. Taking Hall effects in to account Krishna et. al.,[14] have investigated Hall effects on the unsteady hydromagnetic boundary layer flow. Rao et. al., [17] have analyzed Hall effects on unsteady Hydromagnetic flow. Siva Prasad et. al., [26] have studied Hall effects on unsteady MHD free and forced convection flow in a porous rotating channel. Recently Seth et. al., [21] have investigated the effects of Hall currents on heat transfer in a rotating MHD channel flow in arbitrary conducting walls. Sarkar et. al., [22] have analyzed the effects of mass transfer and rotation and flow past a porous plate in a porous medium with variable suction in slip flow region. Anwar Beg et al[3] have discussed unsteady magnetohydrodynamics Hartmann-Couette flow and heat transfer in a Darcian channel with Hall current, ionslip, Viscous and Joule heating effects. Ahmed [1] has discussed the Hall effects on transient flow pas an impulsively started infinite horizontal porous plate in a rotating system.

\section{FORMULATION OF THE PROBLEM:}

We consider a steady hydromagnetic heat and mass transfer flow of a viscous electrically conducting along a porous infinite vertical plate $\mathrm{y}=0$ in a rotating system. The flow is also assumed to be moving with a uniform velocity $\mathrm{U}_{\infty}$, which is in the $\mathrm{x}$-direction, is taken along the plate in the upward direction and the y-axis is normal to it. Initially the plate is at rest, after that the whole system is allowed to rotate with a constant angular velocity $\Omega$ about the y-axis. The temperature and the species concentration at the plate are constantly raised from $\mathrm{T}_{\infty}$ and $\mathrm{C}_{\infty}$ to $\mathrm{T}_{\mathrm{w}}$ and $\mathrm{C}_{\mathrm{w}}$ respectively, where $\mathrm{T}_{\infty}$ and $\mathrm{C}_{\infty}$ are the temperature and species concentration of the uniform flow respectively. A

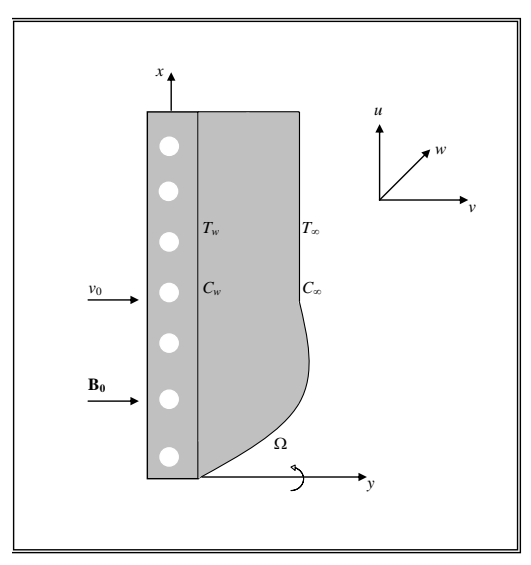

Fig. 1 : Physical configuration and coordinate system uniform magnetic field $\mathrm{B}$ is taken to be along the $\mathrm{y}$-axis which is assumed to be electrically non-conducting. We assumed following (Pai [16]) that the magnetic Reynolds number of the flow is taken to be small enough so that the induced magnetic field is negligible in comparison with applied one, so that $\mathrm{B}=(0, \mathrm{~B} 0,0)$ and the magnetic lines of force are fixed relative to the fluid.

The physical configuration considered here is shown in Figure 1. It is assumed that the plate is semi-infinite in extent and hence all the physical quantities depend on $\mathrm{y}$ and $\mathrm{x}$. Taking hall 
currents into account the basic equations relevant to the problem under Boussinesq's approximation are,

$$
\begin{aligned}
& \frac{\partial u}{\partial x}+\frac{\partial v}{\partial y}=0 \\
& u \frac{\partial u}{\partial x}+v \frac{\partial u}{\partial y}=v \frac{\partial^{2} u}{\partial y^{2}}+\beta g\left(T-T_{\infty}\right)+\beta^{\bullet} g\left(C-C_{\infty}\right)+2 \Omega w+\frac{\sigma B_{o}^{2}}{\rho}\left(U_{o}-u-m w\right)-\left(\frac{v}{k}\right) u \\
& u \frac{\partial w}{\partial x}+v \frac{\partial w}{\partial y}=v \frac{\partial^{2} w}{\partial y^{2}}+2 \Omega\left(U_{o}-u\right)-\frac{\sigma B_{o}^{2}}{\rho}\left(m w-\left(u-U_{o}\right)\right)-\left(\frac{v}{k}\right) w \\
& u \frac{\partial T}{\partial x} v \frac{\partial T}{\partial y}=\frac{k_{f}}{\rho C_{p}} \frac{\partial^{2} T}{\partial y^{2}}+\frac{v}{C_{p}}\left(\left(\frac{\partial u}{\partial y}\right)^{2}+\left(\frac{\partial w}{\partial y}\right)^{2}\right)+ \\
& +\frac{\sigma B_{o}^{2}}{\rho}\left(\left(U_{o}-u\right)^{2}+w^{2}\right) \\
& u \frac{\partial C}{\partial x} v \frac{\partial C}{\partial y}=D_{m} \frac{\partial^{2} C}{\partial y^{2}}+\frac{D_{m} K_{T}}{T_{m}} \frac{\partial^{2} T}{\partial y^{2}}-\gamma^{\prime}\left(C-C_{\infty}\right)
\end{aligned}
$$

The boundary conditions for the problem are

$$
\begin{aligned}
& u=b x, v=-v_{w}, w=0, \frac{\partial T}{\partial y}=-\frac{q_{w}}{k_{f}}, \frac{\partial C}{\partial y}=-\frac{q_{m}}{D_{B}} \quad \text { at } y=0 \\
& u=U_{0} \quad w=0, T \rightarrow T_{\infty}, C \rightarrow C_{\infty} \text { as } y \rightarrow \infty
\end{aligned}
$$

Where $b>0$. The boundary conditions on the velocity in(6) are the no-slip conditions at the surface at $\mathrm{y}=0$, while the boundary conditions on the velocity as $y \rightarrow \infty$ follow from the fact that there is no flow far away from the stretching surface. The temperature and species concentration are maintained at a prescribed constant values $\mathrm{T}_{\mathrm{w}}$ and $\mathrm{C}_{\mathrm{w}}$ at the sheet and are assumed to vanish far away from the sheet.

Following the work of Sattar [20], a transformation is now made as

$$
u_{1}=U_{0}-u \quad \Rightarrow u=U_{0}-u_{1}
$$

Equations (1)-(5) and the boundary conditions (4), respectively, transform to

$$
\begin{aligned}
& -\frac{\partial u_{1}}{\partial x}+\frac{\partial v}{\partial y}=0 \\
& \left(U_{0}-u_{1}\right) \frac{\partial u_{1}}{\partial x}+v \frac{\partial u_{1}}{\partial y}=v \frac{\partial^{2} u_{1}}{\partial y^{2}}-\beta g\left(T-T_{\infty}\right)-\beta^{\bullet} g\left(C-C_{\infty}\right)-\frac{\sigma B_{o}^{2}}{\rho}\left(u_{1}-m w\right)-\left(\frac{v}{k}\right)\left(U_{0}-u_{1}\right) \\
& \left(U_{0}-u_{1}\right) \frac{\partial w}{\partial x}+v \frac{\partial w}{\partial y}=v \frac{\partial^{2} w}{\partial y^{2}}+2 \Omega u_{1}-\frac{\sigma B_{o}^{2}}{\rho}\left(m w-u_{1}\right)-\left(\frac{v}{k}\right) w \\
& \left(U_{0}-u_{1}\right) \frac{\partial T}{\partial x}+v \frac{\partial T}{\partial y}=\frac{k}{\rho C_{p}} \frac{\partial^{2} T}{\partial y^{2}}+\frac{v}{C_{p}}\left(\left(\frac{\partial u_{1}}{\partial y}\right)^{2}+\left(\frac{\partial w}{\partial y}\right)^{2}\right)+ \\
& \quad+\frac{\sigma B_{o}^{2}}{\rho C_{p}}\left(u_{1}^{2}+w^{2}\right) \\
& \left(U_{0}-u_{1}\right) \frac{\partial C}{\partial x}+v \frac{\partial C}{\partial y}=D_{m} \frac{\partial^{2} C}{\partial y^{2}}+\frac{D_{m} k_{T}}{T_{m}} \frac{\partial^{2} T}{\partial y^{2}}-K_{c}^{\prime} C
\end{aligned}
$$




$$
\begin{aligned}
& u_{1}=a x, v=v_{o}(x), w=0, \frac{\partial T}{\partial y}=-\frac{q_{w}}{k_{f}}, \frac{\partial C}{\partial y}=-\frac{q_{m}}{D_{B}} \quad \text { at } y=0 \\
& u_{1}=0 \quad w=0, T \rightarrow T_{\infty}, C \rightarrow C_{\infty} \quad \text { as } y \rightarrow \infty
\end{aligned}
$$

Where $\mathrm{u}, \mathrm{v}, \mathrm{w}$ are the velocity components in the $\mathrm{x}, \mathrm{y}, \mathrm{z}$ directions respectively, $v$ is the kinematics viscosity, $\mathrm{g}$ is the acceleration due to gravity, $\rho$ is the density, $\beta$ is the coefficient of Volumetric thermal expansion, $\beta^{\bullet}$ is the Volumetric mass expansion. $\mathrm{T}, \mathrm{T}_{\mathrm{w}}$, $\mathrm{T}_{\infty}$ are the temperature of the fluid inside the thermal boundary layer, the plate temperature and the fluid temperature in the free stream respectively, while $\mathrm{C}, \mathrm{C}_{\mathrm{w}}, \mathrm{C}_{\infty}$ are the corresponding concentrations. Also, $\mathrm{K}^{1}$ is the permeability of the porous medium. $\mathrm{k}$ is the thermal conductivity of the medium, $\mathrm{Dm}$ is the coefficient of mass diffusivity, $\mathrm{Cp}$ is the specific heat constant pressure, $\mathrm{Tm}$ is the mean fluid temperature, $\mathrm{k}_{\mathrm{T}}$ is the thermal diffusion ratio, $\mathrm{Cp}$ is the concentration and other symbols have their usual meaning, $\mathrm{C}_{\mathrm{s}}$ is the concentration susceptibility and other symbols have their usual meaning.

\section{MATHEMATICAL ANALYSIS:}

In order to solve equations (9)-(12) under the boundary conditions (13), we adopt the welldefined similarity analysis to attain similarity solutions.

For this purpose, the following similarity transformations are now introduced:

$$
\begin{aligned}
& \eta=y \sqrt{\frac{U_{o}}{2 v x}}, g_{o}(\eta)=\frac{w}{U_{o}}, \theta(\eta)=\frac{T-T_{\infty}}{T_{w}-T_{\infty}}, \phi(\eta)=\frac{C-C_{\infty}}{C_{w}-C_{\infty}}, \psi=\sqrt{2 v x U_{0}} f(\eta) \\
& u_{1}=\frac{\partial \psi}{\partial y}=U_{o} f^{\prime}(\eta) \mathrm{m} \frac{u}{U_{0}}==1-f^{\prime}(\eta)
\end{aligned}
$$

Now for reasons of similarity, the plate of concentration is assumed to be

$$
C_{w}(x)=C_{o}+\stackrel{\omega}{x}\left(C_{0}-C_{\infty}\right)
$$

where $C_{0}$ is considered to be mean concentration and $\underset{x}{\mathbb{x}}=\frac{x U_{o}}{v}$

The continuity equation (1) then yields

$$
v=\frac{\partial \psi}{\partial x}=-\sqrt{\frac{v U_{o}}{2 x}\left(\eta f^{\prime}(\eta)-f(\eta)\right)}
$$

Also we have $f_{w}=v_{W}(x) \sqrt{\frac{2 x}{v U_{0}}}$

Where $f_{w}$ is the suction parameter or transpiration parameter and clearly in (16) $f_{w}<0$ corresponds to suction and $f_{w}>0$ corresponds to injection at the plate. From equations (9)-(13) and (14)-(14), we have the following dimensionless ordinary coupled non-linear differential equations.

$$
\begin{aligned}
& f^{\prime \prime \prime}+(\eta-f) f^{\prime \prime}-G r(\theta+N \phi)-\left(M^{2}+D^{-1}\right) f^{\prime}-\frac{M^{2}}{1+m^{2}}\left(f^{\prime}+m g\right)=0 \\
& g_{0}^{\prime \prime}+(\eta-f) g_{0}^{\prime}+R f^{\prime}-M^{2} g_{0}=0 \\
& \theta^{\prime \prime}+P(\eta-f) \theta^{\prime}+P E_{c}\left(\left(f^{\prime \prime}\right)^{2}+\left(g_{o}^{\prime}\right)^{2}\right)+P E_{c} M\left(\left(f^{\prime}\right)^{2}=0\right. \\
& \phi^{\prime \prime}+S c(\eta-f) \phi^{\prime}+2 S c\left(f^{1}-(1+\gamma)\right) \phi+S c S_{r} \theta^{\prime \prime}=0
\end{aligned}
$$

With the corresponding boundary conditions 


$$
\begin{aligned}
& f=f_{w,} f^{\prime}=1, g_{0}=0, \frac{d \theta}{d \eta}=-1, \frac{d \phi}{d \eta}=-1 \quad \text { at } \eta=0 \\
& f^{\prime}=0 \quad g_{0}=0, \theta=0, \varphi=0 \text { as } \eta \rightarrow \infty
\end{aligned}
$$

where

$G_{r}=\frac{2 \beta g\left(T_{w}-T_{\infty}\right) x^{3}}{v^{2}}($ GrashofNumber $), N=\frac{\beta^{\bullet}\left(C_{w}-C_{\infty}\right)}{\left(T_{w}-T_{\infty}\right)}($ Buouyancyp parameter $)$

$D^{-1}=\frac{2 v x}{K U_{0}}($ InverseDarcy parameter $), M=\frac{2 x \sigma B_{0}^{2}}{\rho U_{0}}($ Magnetic parameter $)$,

$M_{1}^{2}=\frac{M^{2}}{1+m^{2}}, R=\frac{4 \Omega x}{U_{0}}($ Rotational parameter $), P_{r}=\frac{\rho v C_{p}}{k}(\operatorname{Pr}$ andtl Number $)$,

$E_{c}=\frac{U_{0}^{2}}{C_{p}\left(T_{w}-T_{\infty}\right)}($ Ecker tnumber $), S c=\frac{v}{D_{m}}($ SchmidtNumber $)$,

So $=\frac{D_{m} k_{T}\left(T_{w}-T_{\infty}\right)}{C_{p} C_{s}\left(C_{w}-C_{\infty}\right)}($ Soret parameter $), \gamma=\frac{k_{c}\left(C_{w}-C_{\infty}\right)}{U_{0}}($ Chemical reaction parameter $)$

For the computational purpose and without loss of generality $\infty$ has been fixed as 8 . The whole domain is divided into 11 line elements of equal width, each element being three nodded.

\section{FINITE ELEMENT ANALYSIS}

The method basically involves the following steps:

(1) Division of the domain into elements, called the finite element mesh.

(2) Generation of the element equations using variational formulations.

(3) Assembly of element equations as in step 2.

(4) Imposition of boundary conditions to the equations obtained in step 3

(5) Solution of the assumed algebraic equations.

The local stiffness matrices obtained are assembled into global matrix using inter element continuity and equilibrilium conditions and boundary conditions. The resulting matrices are solved employing iteration process.

\section{Skin friction coefficient, Nusselt Number and Sherwood Number:}

The quantities of chief physical interest are the skin friction coefficients, the Nusselt Number and the Sherwood number. The wall skin frictions are defined by

$$
\begin{aligned}
& \tau_{x}=\mu\left(\frac{\partial u}{\partial y}\right)_{y=0} \text { and } \tau_{z}=\mu\left(\frac{\partial w}{\partial y}\right)_{y=0} \text { which are proportional to } \\
& \left(\frac{\partial^{2} f}{\partial \eta^{2}}\right)_{\eta=0} \text { and }\left(\frac{\partial g_{0}}{\partial \eta}\right)_{\eta=0}
\end{aligned}
$$

The Nusselt Number is defined by $N u=\frac{1}{\Delta T}\left(\frac{\partial T}{\partial y}\right)_{y=0}$ which is proportional to $\left(\frac{\partial \theta}{\partial \eta}\right)_{\eta=0}$ The Sherwood Number is defined by $S h=\frac{1}{\Delta C}\left(\frac{\partial C}{\partial y}\right)_{y=0}$ which is proportional to $\left(\frac{\partial \phi}{\partial \eta}\right)_{\eta=0}$

The numerical values of the skin friction coefficients, the Nusselt Number and the Sherwood Number are sorted in table .2 . 


\section{COMPARISON:}

In the absence of Hall currents $(m=0)$ and no slip regime $(A=0)$ the results are in good agreement with Sreerangavani [27].

Table.1 shows the comparison

\begin{tabular}{|l|l|l|l|l|l|l|l|l|}
\hline \multicolumn{3}{|l|}{ Parameters } & \multicolumn{3}{l|}{ SrirangaVani et al [27] } & \multicolumn{3}{l|}{ Present results(m=0,A=0) } \\
\hline $\mathbf{R}$ & So & $\mathbf{E c}$ & $\boldsymbol{\tau}(\mathbf{0})$ & $\mathbf{N u}(\mathbf{0})$ & $\mathbf{S h}(\mathbf{0})$ & $\boldsymbol{\tau}(\mathbf{0})$ & $\mathbf{N u}(\mathbf{0})$ & $\mathbf{S h}(\mathbf{0})$ \\
\hline $\mathbf{0 . 5}$ & $\mathbf{0 . 5}$ & $\mathbf{0 . 0 1}$ & 3.49064 & 0.07426 & 1.2087 & 3.49061 & 0.07423 & 1.2089 \\
\hline $\mathbf{1 . 0}$ & $\mathbf{0 . 5}$ & $\mathbf{0 . 0 1}$ & 3.48376 & $\mathbf{0 . 0 7 3 2 8}$ & 1.2079 & 3.48374 & $\mathbf{0 . 0 7 3 2 6}$ & 1.2079 \\
\hline $\mathbf{1 . 5}$ & $\mathbf{0 . 5}$ & $\mathbf{0 . 0 1}$ & 3.47259 & $\mathbf{0 . 0 7 1 6 4}$ & 1.2054 & 3.47257 & $\mathbf{0 . 0 7 1 6 3}$ & 1.2056 \\
\hline $\mathbf{0 . 5}$ & $\mathbf{1 . 0}$ & $\mathbf{0 . 0 1}$ & 3.48853 & 0.07523 & 1.2761 & 3.48855 & 0.07524 & 1.2760 \\
\hline $\mathbf{0 . 5}$ & $\mathbf{1 . 5}$ & $\mathbf{0 . 0 1}$ & 3.48704 & 0.07596 & 1.3214 & 3.48702 & 0.07595 & 1.3212 \\
\hline $\mathbf{0 . 5}$ & $\mathbf{0 . 5}$ & $\mathbf{0 . 0 3}$ & 3.43136 & 0.08732 & 1.4987 & 3.43139 & 0.08733 & 1.4984 \\
\hline $\mathbf{0 . 5}$ & $\mathbf{0 . 5}$ & $\mathbf{0 . 0 5 5}$ & 3.57222 & 0.05684 & 0.8670 & 3.57226 & 0.05684 & 0.8672 \\
\hline $\mathbf{0 . 5}$ & $\mathbf{0 . 5}$ & $\mathbf{0 . 0 7}$ & 3.69426 & 0.03131 & 0.4445 & 3.69427 & 0.03130 & 0.4446 \\
\hline
\end{tabular}

\section{DISCUSSION OF THE NUMERICAL RESULTS:}

The non-linear linear equations governing the flow have been analysed by employing Galerkin finite element technique with three nodded line segments. The velocity, temperature and concentrations distributions have been analysed for different variations of the parameters $\mathrm{m}, \mathrm{Sc}, \mathrm{Ec}, \mathrm{R}, \mathrm{A}$ and $\mathrm{S}_{\mathrm{o}}$.

Figs.2a-7a show the variation of the axial velocity $\mathrm{fl}(\eta)$ with different values of $\mathrm{m}, \mathrm{Sc}$, Ec, $R, A$ and $S_{0}$ An increase in the Hall parameter(m) increases the axial velocity in the flow region. Lesser the molecular diffusivity larger the velocity. Increasing the Soret parameter $\mathrm{S}_{0}$ larger the axial velocity in the flow region. With reference to Ec, it can be seen that higher the dissipative heat larger the velocity. Thus the presence of the dissipative term leads to an enhancement in the axial velocity. Represent $f^{1}(\eta)$ with rotation parameter R. It can observed from the profiles that $f^{1}(\eta)$ enhances with increase in the rotation parameter R.

Figs. $2 b-7 b$ show the cross velocity $g(\eta)$ ) which arises due to the rotation is shown in figures for different parametric values. It is found that the cross velocity $g(\eta)$ enhances with increase in $\mathrm{m}, \mathrm{Sc}, \mathrm{Ec}, \mathrm{R}, \mathrm{A}$ and reduces with Soret parameter and slip parameter A. When the molecular buoyancy force dominates over the thermal buoyancy force the magnitude of cross velocity reduces when the buoyancy forces are in the same direction and for the forces acting in opposite directions, it increases in the flow region.

The non-dimensional temperature $(\theta)$ is shown in figures for different parametric values. Higher the Hall parameter $(\mathrm{m})$ /lesser permeability of the porous medium smaller the temperature in the flow region. With reference to $\mathrm{Sc}$, we notice a depreciation in the temperature with increase in $\mathrm{Sc} \leq 0.66$ and for higher $\mathrm{Sc} \geq 1.3$, we notice an enhancement in the temperature. Increasing the Soret parameter $S_{0}$ results in a depreciation in the temperature. An increase in rotation parameter R / Eckert number Ec leads to an enhancement in the temperature (figs.2c-7c).

The concentration distribution $(\mathrm{C})$ is shown in figures for different parametric values. We follow the convention that the non-dimensional concentration is positive/negate according as actual concentration is greater/lesser than the ambient concentration. We find that the actual concentration enhances with Sc. Increasing Soret parameter $S_{0}$ leads to an enhancement in the concentration. We find that the concentration reduces with Eckert number $\mathrm{E}$ and slip parameter (A). An increase in rotation parameter $\mathrm{R}$ leads to an enhancement in the concentration (figs.2d-7d). 


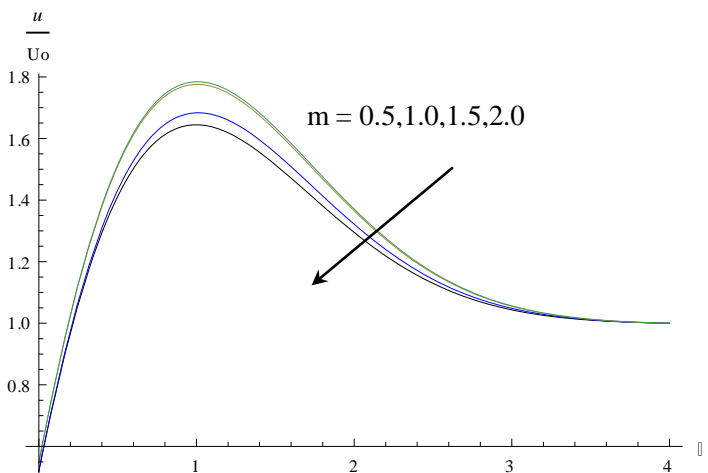

Fig.2a Variation of $\mathrm{u} / \mathrm{Uo}$ with $\mathrm{m}$

$\mathrm{Sc}=1.3, \mathrm{So}=0.5, \mathrm{~A}=0.2, \mathrm{Ec}=0.01, \mathrm{R}=0.5$

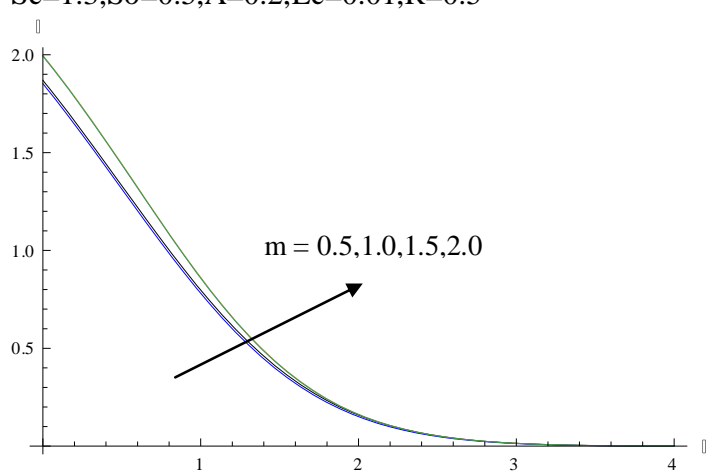

Fig.2c Variation of $\theta$ with $m$

$\mathrm{Sc}=1.3, \mathrm{So}=0.5, \mathrm{~A}=0.2, \mathrm{Ec}=0.01, \mathrm{R}=0.5$

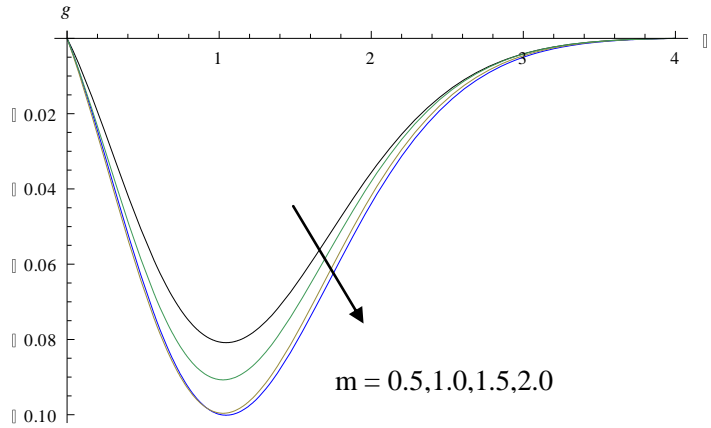

Fig.2b Variation of $\mathrm{g}$ with $\mathrm{m}$

$\mathrm{Sc}=1.3, \mathrm{So}=0.5, \mathrm{~A}=0.2, \mathrm{Ec}=0.01, \mathrm{R}=0.5$

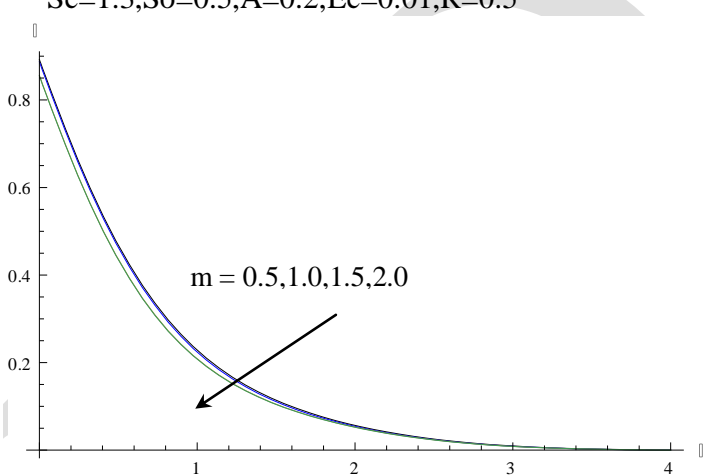

Fig.2d Variation of $\phi$ with $m$

$\mathrm{Sc}=1.3, \mathrm{So}=0.5, \mathrm{~A}=0.2, \mathrm{Ec}=0.01, \mathrm{R}=0.5$

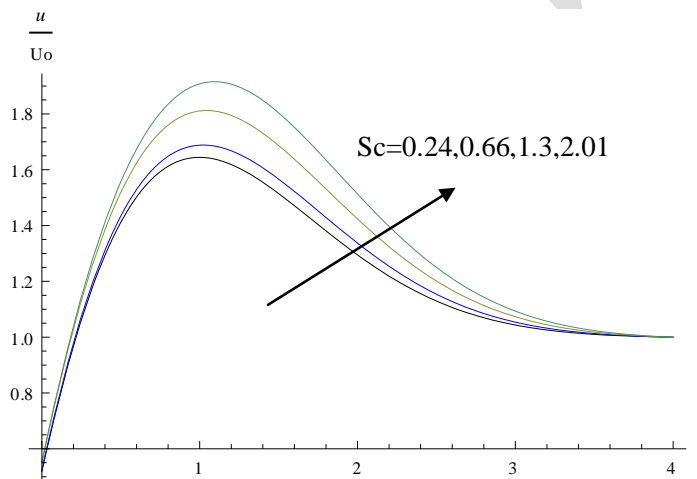

Fig.3a Variation of $\mathrm{u} / \mathrm{Uo}$ with Sc

$\mathrm{m}=0.5, \mathrm{So}=0.5, \mathrm{~A}=0.2, \mathrm{Ec}=0.01, \mathrm{R}=0.5$
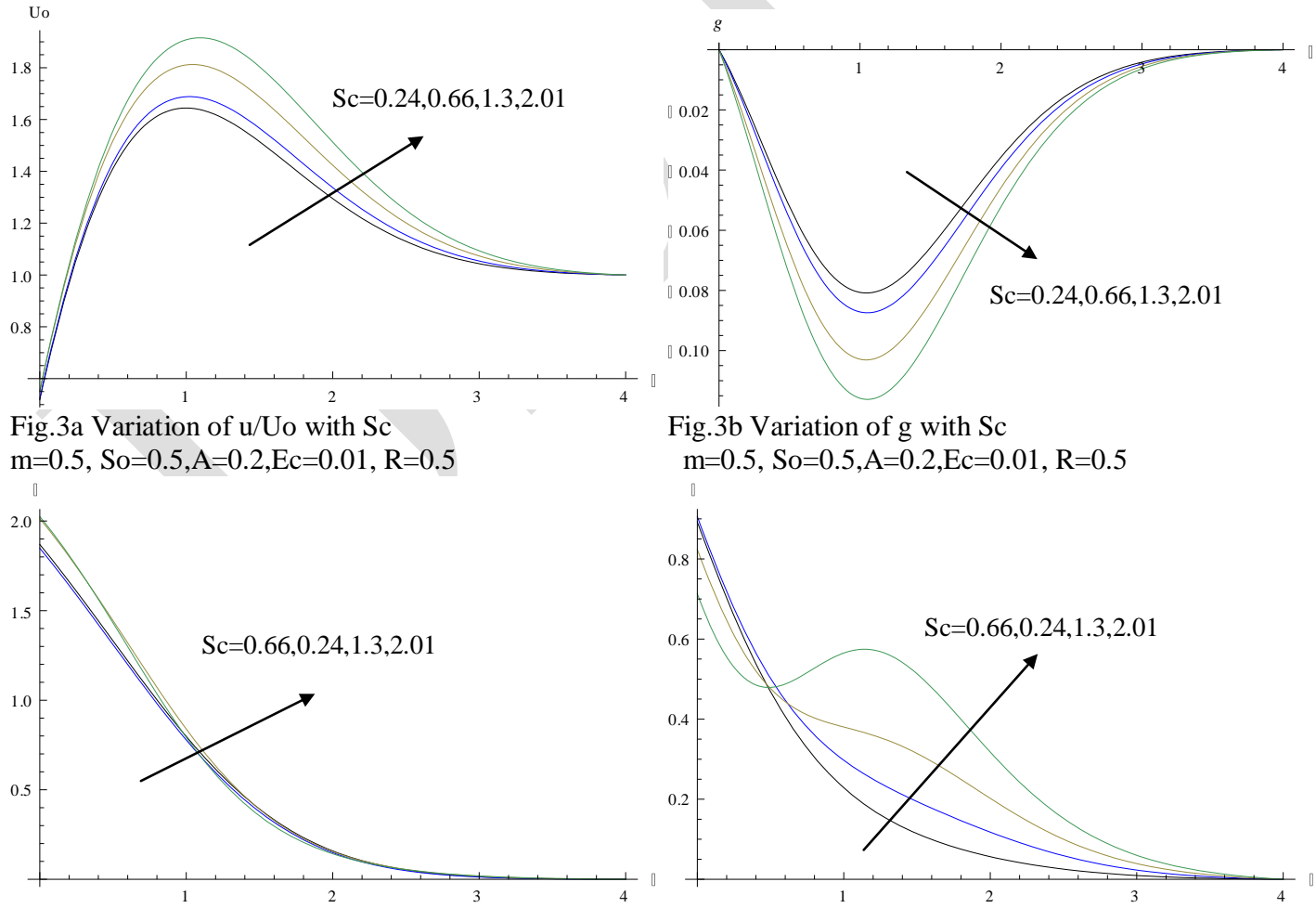

Fig.3c Variation of $\theta$ with Sc $\mathrm{m}=0.5$, So $=0.5, \mathrm{~A}=0.2, \mathrm{Ec}=0.01, \mathrm{R}=0.5$

Fig.3b Variation of $\mathrm{g}$ with $\mathrm{Sc}$ $\mathrm{m}=0.5, \mathrm{So}=0.5, \mathrm{~A}=0.2, \mathrm{Ec}=0.01, \mathrm{R}=0.5$

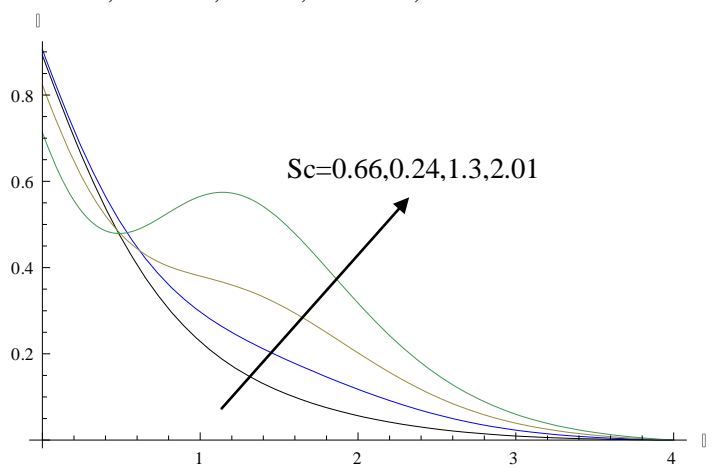

Fig.3d Variation of $\phi$ with $\mathrm{Sc}$ $\mathrm{m}=0.5, \mathrm{So}=0.5, \mathrm{~A}=0.2, \mathrm{Ec}=0.01, \mathrm{R}=0.5$ 


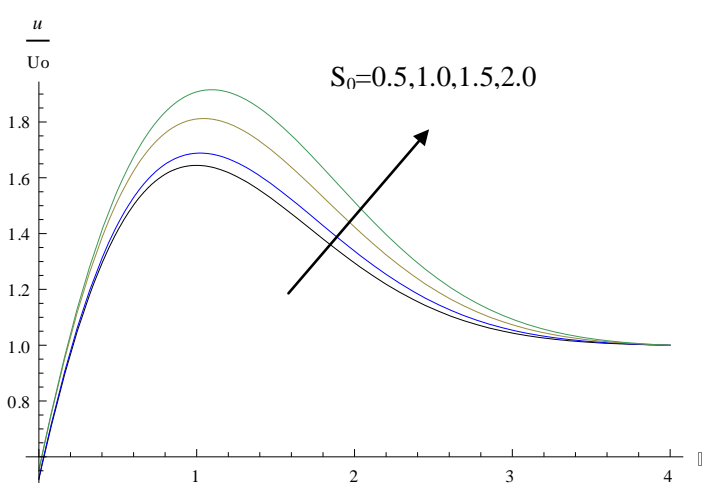

Fig.4a Variation of $\mathrm{u} / \mathrm{Uo}$ with So $\mathrm{m}=0.5, \mathrm{~A}=0.2, \mathrm{Ec}=0.01, \mathrm{R}=0.5, \mathrm{Sc}=1.3$
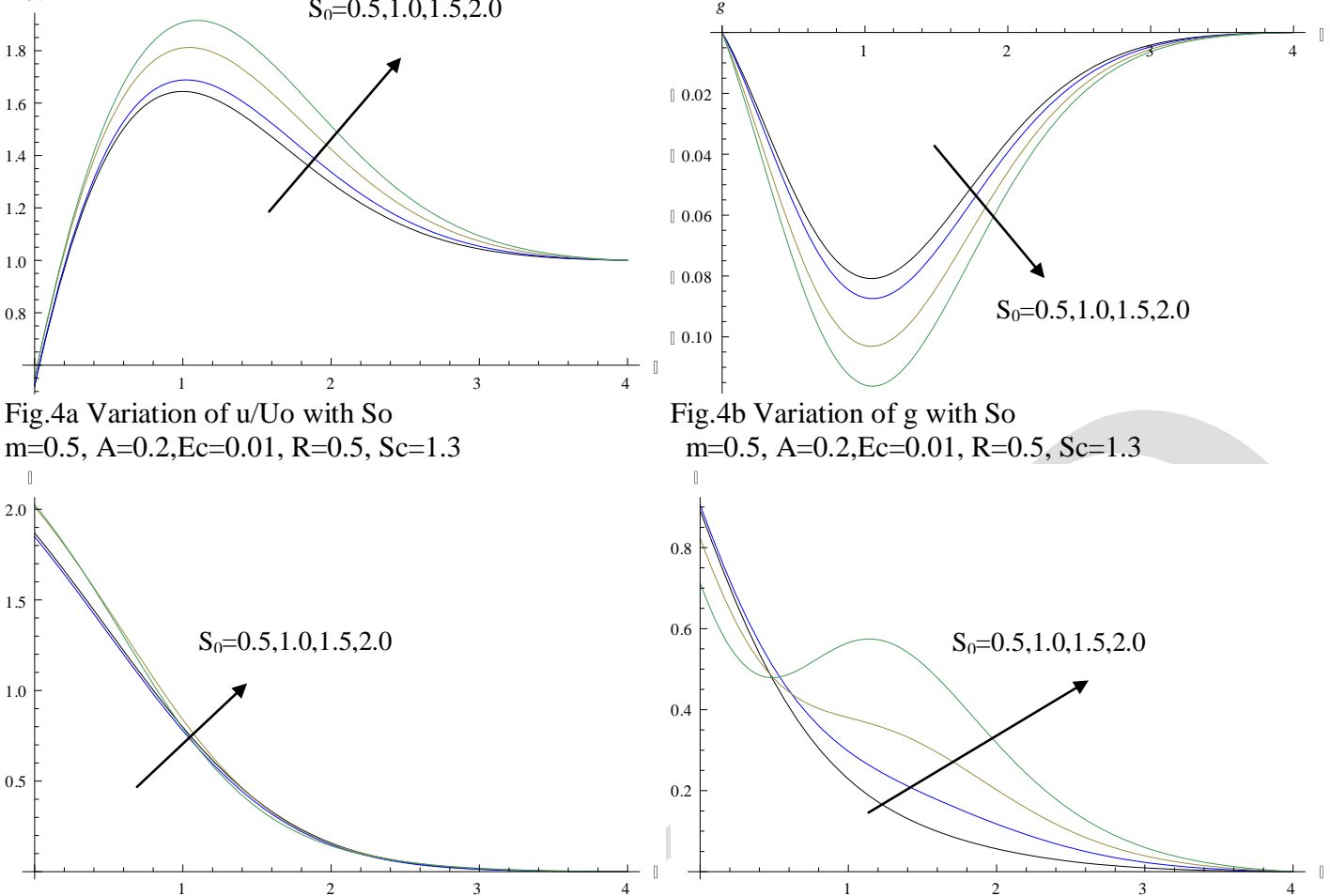

Fig.4c Variation of $\theta$ with So

Fig. $4 \mathrm{~b}$ Variation of $\mathrm{g}$ with So $\mathrm{m}=0.5, \mathrm{~A}=0.2, \mathrm{Ec}=0.01, \mathrm{R}=0.5, \mathrm{Sc}=1.3$

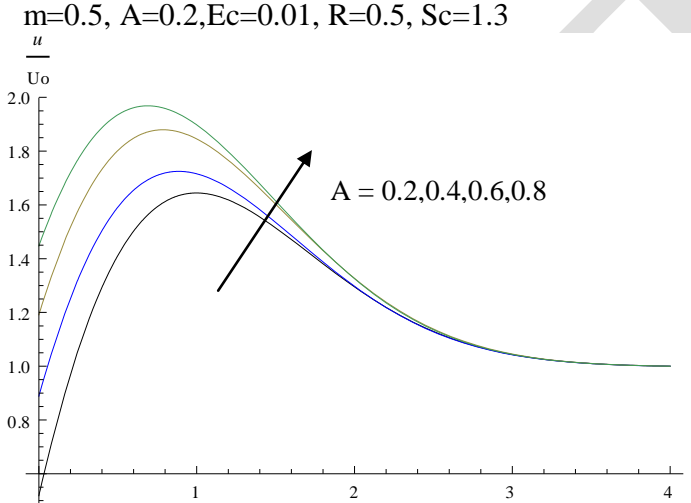

Fig.5a Variation of $\mathrm{u} / \mathrm{Uo}$ with A $\mathrm{m}=0.5, \mathrm{Sc}=1.3, \mathrm{So}=0.5, \mathrm{Ec}=0.01, \mathrm{R}=0.5$

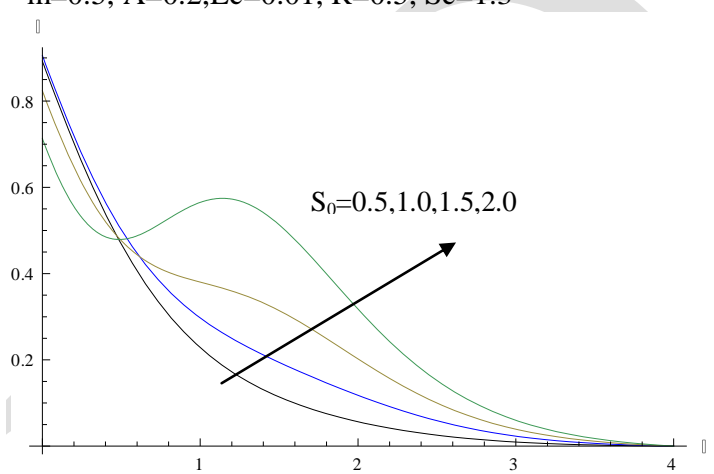

Fig.4d Variation of $\phi$ with So $\mathrm{m}=0.5, \mathrm{~A}=0.2, \mathrm{Ec}=0.01, \mathrm{R}=0.5, \mathrm{Sc}=1.3$
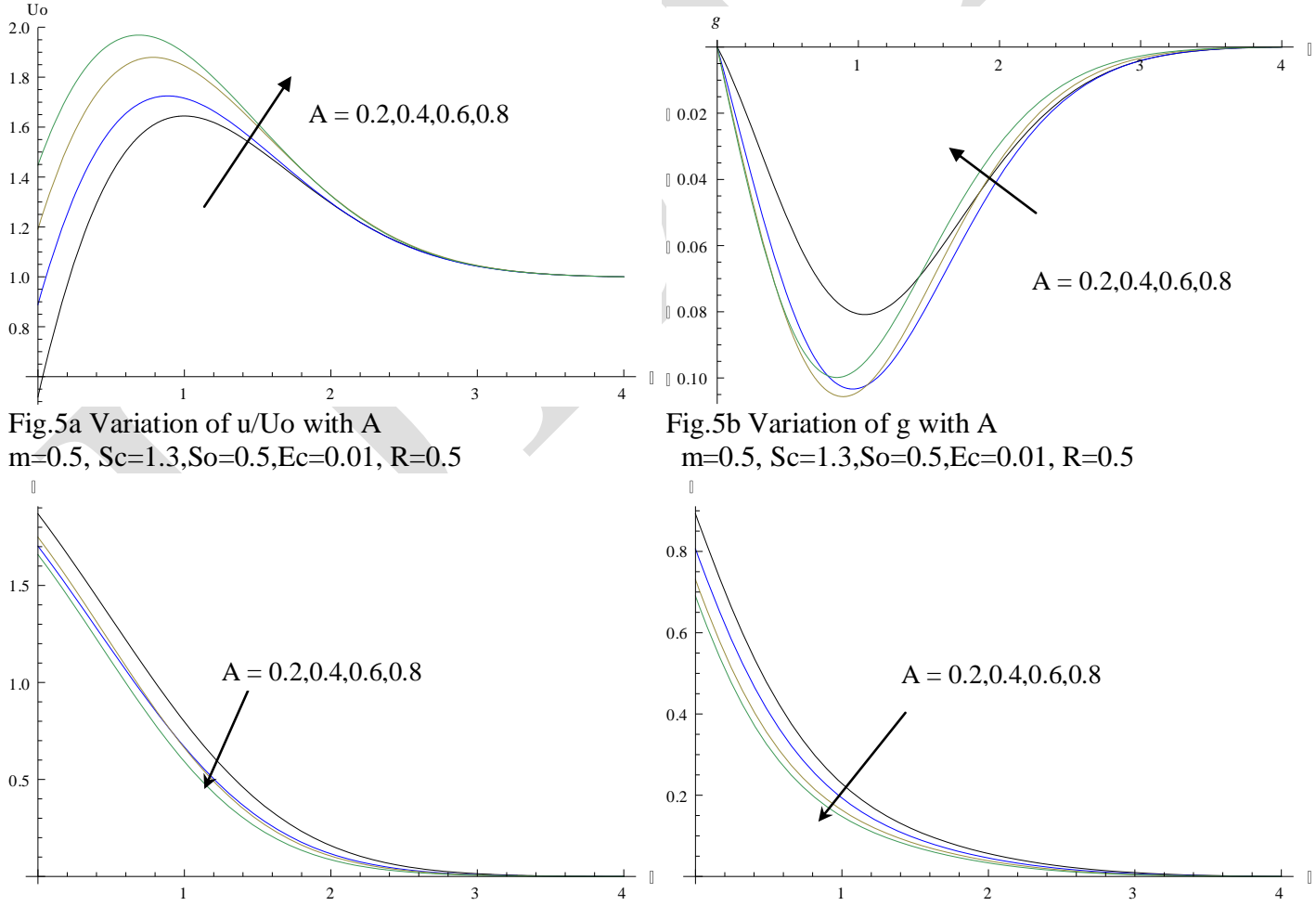

Fig.5c Variation of $\theta$ with A

Fig.5b Variation of $g$ with A $\mathrm{m}=0.5, \mathrm{Sc}=1.3, \mathrm{So}=0.5, \mathrm{Ec}=0.01, \mathrm{R}=0.5$

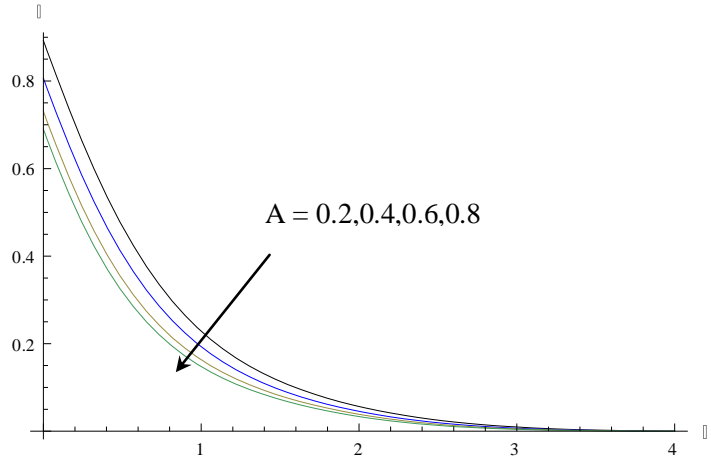

$\mathrm{m}=0.5, \mathrm{Sc}=1.3, \mathrm{So}=0.5, \mathrm{Ec}=0.01, \mathrm{R}=0.5$

Fig.5d Variation of $\phi$ with A $\mathrm{m}=0.5, \mathrm{Sc}=1.3, \mathrm{So}=0.5, \mathrm{Ec}=0.01, \mathrm{R}=0.5$ 


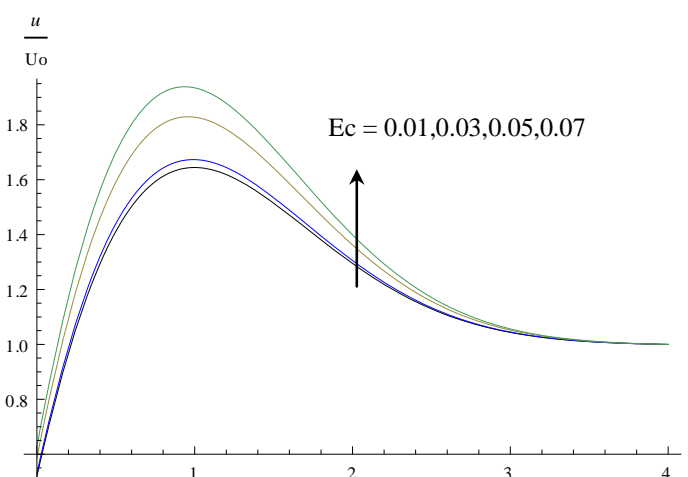

Fig.6a Variation of u/Uo with Ec $\mathrm{m}=0.5, \mathrm{Sc}=1.3, \mathrm{So}=0.5, \mathrm{~A}=0.2, \mathrm{R}=0.5$
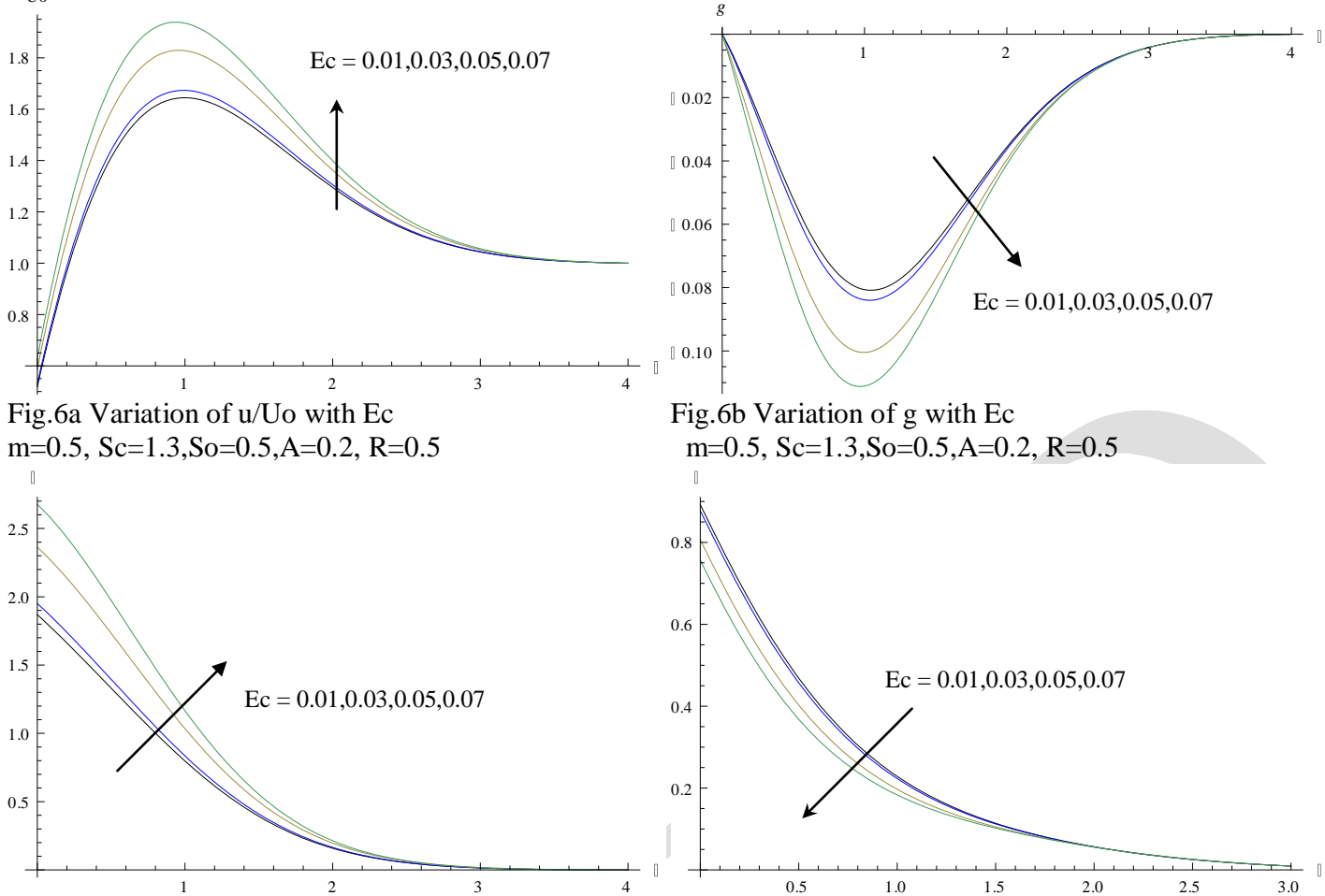

Fig.6b Variation of $\mathrm{g}$ with Ec $\mathrm{m}=0.5, \mathrm{Sc}=1.3, \mathrm{So}=0.5, \mathrm{~A}=0.2, \mathrm{R}=0.5$

Fig.6c Variation of $\theta$ with Ec

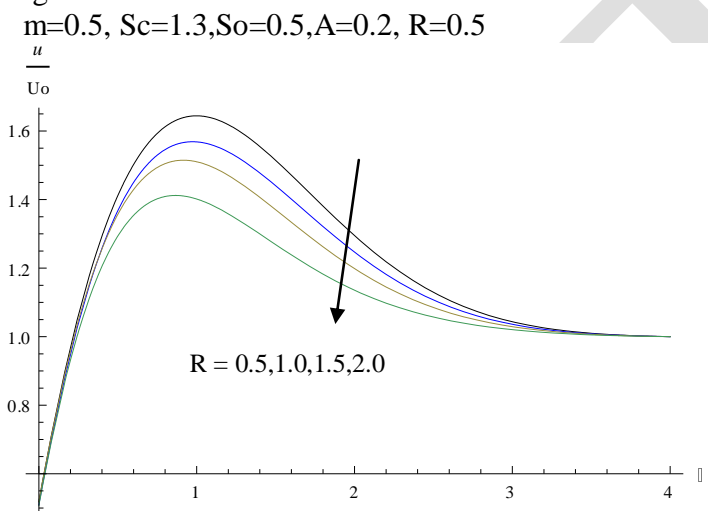

Fig.6d Variation of $\phi$ with Ec $\mathrm{m}=0.5, \mathrm{Sc}=1.3, \mathrm{So}=0.5, \mathrm{~A}=0.2, \mathrm{R}=0.5$
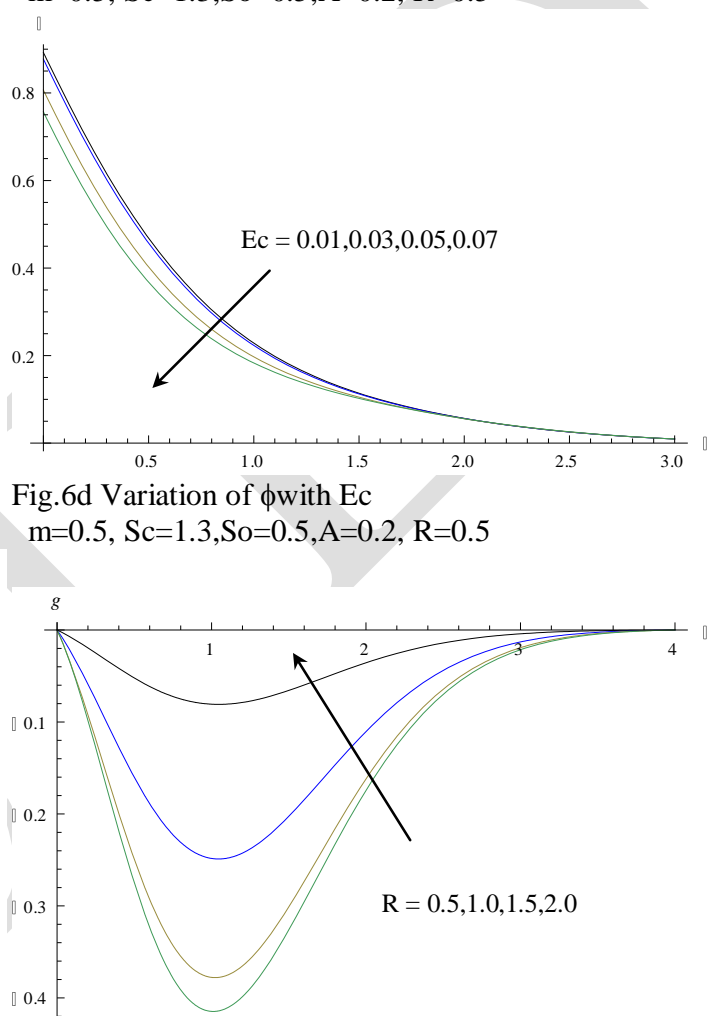

Fig.7a Variation of $\mathrm{u} / \mathrm{Uo}$ with $\mathrm{R}$ $\mathrm{m}=0.5, \mathrm{Sc}=1.3, \mathrm{So}=0.5, \mathrm{Ec}=0.01, \mathrm{~A}=0.2$

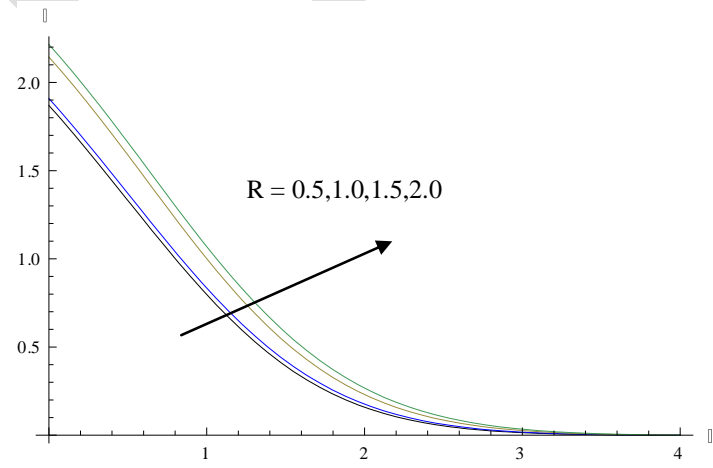

Fig.7c Variation of $\theta$ with $\mathrm{R}$ $\mathrm{m}=0.5, \mathrm{Sc}=1.3, \mathrm{So}=0.5, \mathrm{Ec}=0.01, \mathrm{~A}=0.2$

Fig.7b Variation of $\mathrm{g}$ with $\mathrm{R}$ $\mathrm{m}=0.5, \mathrm{Sc}=1.3, \mathrm{So}=0.5, \mathrm{Ec}=0.01, \mathrm{~A}=0.2$

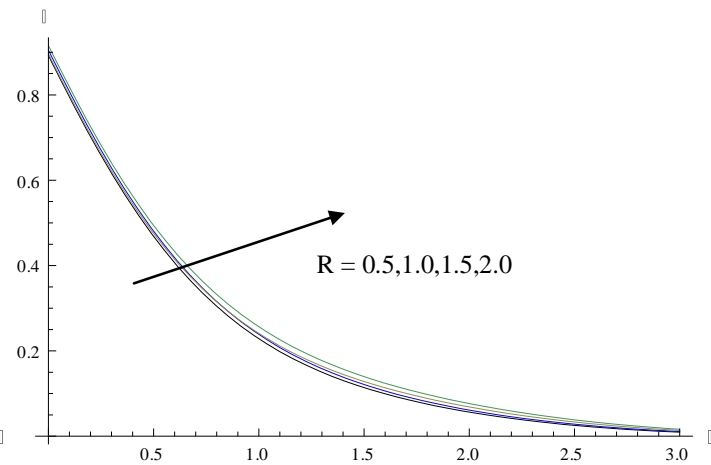

Fig.7d Variation of $\phi$ with $\mathrm{R}$ $\mathrm{m}=0.5, \mathrm{Sc}=1.3, \mathrm{So}=0.5, \mathrm{Ec}=0.01, \mathrm{~A}=0.2$

The components of skin friction $\tau_{\mathrm{x}} \& \tau_{\mathrm{z}}$ are depicted in tables 2 for different values of $\mathrm{m}, \mathrm{A}, \mathrm{Sc}, \mathrm{R}, \mathrm{Ec}$ and $\mathrm{S}_{0}$. We find that when the moleular buoyancy force dominates over the 
thermal buoyancy force $\tau_{\mathrm{x}}$ and $\tau_{\mathrm{z}}$ enhances at $\eta=0$ when the buoyancy forces are in the same direction and for the forces acting in opposite directions $\tau_{\mathrm{x}}$ and $\tau_{\mathrm{z}}$ reduces at $\eta=0$. An increase in Sc leads to a decrease in $\tau_{\mathrm{x}}$ and $\tau_{\mathrm{z}}$ at $\eta=0$. The variation of $\tau_{\mathrm{x}}$ and enhances $\tau_{\mathrm{z}}$ with rotation parameter $\mathrm{R}$ shows that an increase in $\mathrm{R} \leq 1.0$ reduces $\tau_{\mathrm{x}}$ and enhances $\tau_{\mathrm{z}}$ at the wall and for higher $\mathrm{R} \geq 1.5$. $\tau_{\mathrm{x}}$ and $\tau_{\mathrm{z}}$ enhances at the wall. It can be seen that increasing the Soret parameter $S_{0}$ results in an enhancement in $\tau_{x}$ and $\tau_{z}$ at the wall $\eta=0$.

Table.2 Skin friction components,Nusselt number and Sherwood number

\begin{tabular}{|cr|c|c|c|c|}
\hline \multicolumn{2}{|c|}{ Parameters } & $\tau_{x}(\mathbf{0})$ & $\boldsymbol{\tau}_{z}(\mathbf{0})$ & $\mathbf{N u}(\mathbf{0})$ & $\mathbf{S h}(\mathbf{0})$ \\
\hline $\mathbf{m}$ & $\mathbf{0 . 5}$ & -2.59033 & -0.079692 & 0.534913 & 1.1203 \\
& $\mathbf{1 . 0}$ & -2.60106 & -0.102789 & 0.539857 & 1.12706 \\
& $\mathbf{1 . 5}$ & -2.72838 & -0.111423 & 0.500852 & 1.16808 \\
& $\mathbf{2 . 0}$ & -2.73211 & -0.101966 & 0.501873 & 1.16965 \\
\hline Sc & $\mathbf{0 . 2 4}$ & -2.81527 & -0.113262 & 0.562553 & 0.82456 \\
& $\mathbf{0 . 6 6}$ & -2.59033 & -0.079692 & 0.534913 & 1.12033 \\
& $\mathbf{1 . 3 0}$ & -2.56986 & -0.077108 & 0.473865 & 1.59687 \\
& $\mathbf{2 . 0 1}$ & -2.52956 & -0.0726101 & 0.468042 & 1.82158 \\
\hline $\mathbf{R}$ & $\mathbf{0 . 5}$ & -2.59033 & -0.079692 & 0.534913 & 1.1203 \\
& $\mathbf{1 . 0}$ & -2.58413 & -0.120678 & 0.533361 & 1.11811 \\
& $\mathbf{1 . 5}$ & -2.69052 & -0.185135 & 0.490127 & 1.15165 \\
& $\mathbf{2 . 0}$ & -2.65772 & -0.282248 & 0.482666 & 1.14009 \\
\hline So & $\mathbf{0 . 5}$ & -2.59033 & -0.079692 & 0.534913 & 1.1203 \\
& $\mathbf{1 . 0}$ & -2.64864 & -0.0983021 & 0.548881 & 1.0827 \\
& $\mathbf{1 . 5}$ & -2.75688 & -0.126451 & 0.493793 & 1.40159 \\
& $\mathbf{2 . 0}$ & -2.73041 & -0.138365 & 0.480923 & 2.18381 \\
\hline Ec & $\mathbf{0 . 0 1}$ & -2.59033 & -0.079692 & 0.534913 & 1.12033 \\
& $\mathbf{0 . 0 3}$ & -2.64414 & -0.086250 & 0.512556 & 1.13957 \\
& $\mathbf{0 . 0 5}$ & -2.82623 & -0.109015 & 0.452638 & 1.20124 \\
& $\mathbf{0 . 0 7}$ & -4.12237 & -0.253574 & 0.241422 & 1.77627 \\
\hline A & $\mathbf{0 . 5}$ & -2.59033 & -0.079692 & 0.534913 & 1.1203 \\
& $\mathbf{1 . 0}$ & -2.09003 & -0.123859 & 0.580273 & 1.22909 \\
& $\mathbf{1 . 5}$ & -1.86882 & -0.171669 & 0.55925 & 1.34483 \\
& $\mathbf{2 . 0}$ & -1.63597 & -0.195539 & 0.579651 & 1.40001 \\
\hline
\end{tabular}

The rate of heat transfer (Nusselt number) at $\eta=0$ is exhibited in tables 2 for different parametric values. Higher the thermal buoyancy force /Slip parameter (A) larger the rate of heat transfer at the wall $\eta=0$. An increase in the Hall parameter $m \leq 1.0$,larger the rate of heat transfer and for higher $\mathrm{m} \geq 1.5$,smaller the Nusselt number at the wall. $|\mathrm{Nu}|$ reduces with increase in the rotation parameter $\mathrm{R}$ or Eckert number Ec. Increasing the Soret parameter So $\leq 1.0$ and for higher $\mathrm{So} \geq 1.5$, we notice a depreciation in $\mathrm{Nu}$ at the wall.

The rate of mass transfer (Sherwood number) at $\eta=0$ is shown in tables 2 for different parametric values. It is found that the rate of mass transfer enhances with increase in $\mathrm{m}, \mathrm{Sc}$ and $\mathrm{A}$. $|\mathrm{Sh}|$ reduces with increase in the rotation parameter $\mathrm{R}$ and enhances with increase in Ec. Increasing the Soret parameter $S_{0}$ leads to an enhancement in the rate of mass transfer at the wall. 


\section{CONCLUSIONS :}

An attempt has been made to discuss the combined impact of rotation and Hall currents on convective heat and mass transfer flow of a viscous fluid through a porous medium past a stretching surface. Using Finite element technique the governing equations have been solved .The important conclusion of this analysis are

2) An increase in Hall parameter enhances the velocities and reduces the temperature and concentration. The stress components, Nusselt and Sherwood number enhances with $\mathrm{m}$.

3) An increase in rotation parameter ( $R$ ) reduces the primary velocity and enhances the secondary velocity, temperature and concentration in the flow region.

4) The effect of thermo-diffusion is to enhance the velocities, concentration and reduces the temperature. The stress components, temperature enhances while the concentration reduces with So.

\section{REFERENCES:}

[1] Ahmed N and H.K. Sarmah : MHD Transient flow past an impulsively started infinite horizontal porous plate in a rotating system with hall current: Int J. of Appl. Math and Mech. 7(2) : 1-15, 2011.

[2] Alam, M.M and Sattar,M.A :Unsteady free convection and mass transfer flow in a rotating system with Hall currents, viscous dissipation and Joule heating ., Joural of Energy heat and mass transfer,V.22,pp.31-39(2000)

[3] Anwar Beg, O,Joaquin Zueco and Takhar,H.S:Unsteady magneto-hydrodynamic Hartmann-Couette flow and heat transfer in a Darcian channel with hall currents, ionslip, Viscous and Joule heating:Network Numerical solutions, Commun Nonlinear Sci Numer Simulat,V.14,pp.1082-1097(2009).

[4] Agarwal, R.S and Dhanapal, C : Numerical solution to the flow of a micro polar fluid flow through porous walls of different permeability. pp. 325-336 (1987).

[5] Balasubramanyam M : Effect of radiation on convective Heat and Mass transfer flow in a horizontal rotating channel communicated to Research India Publications, India (2010).

[6] Chenna Kesavaiah, D, Satyanarayana, P.V and Venkataramana, S : Effects of the chemical reaction and radiation absorption on an unsteady MHD convective heat and mass transfer flow past a semi-infinite vertical permeable moving plate embedded in a porous medium with heat source and suction. Int. J. of Appl. Math. \& Mech., V.7, No.1, pp.52-69 (2011).

[7] Circar and Mukharjee : Effects of mass transfer and rotation on flow past a porous plate in a porous medium with variable suction in slip flow. Acta Cienica Indica, V.34M, No.2, pp.737-751 (2008).

[8] Debnath,L:Exact solutions of unsteady hydrodynamic and hydromagnetic boundary layer equations in a rotating fluid system, ZAMM, V.55, p.431 (1975)

[9] El.Mistikawy, T.M.A, Attia, H.A : The rotating disk flow in the presence of Strong magnetic field. Proc. $3^{\text {rd }}$ Int. Congr. of fluid mechanics. Cairo, Egypt. V.3, 2-4 January, pp 1211-1222 (1990).

[10] Ghouse, S.K : A note on steady and unsteady hydro magnetic flow in rotating channel in the presence of inclined magnetic field. Int. J. Eng. Sci., V.29, No.8, pp.10131016(1991).

[11] Hazem Ali Attia : Unsteady MHD flow near a rotating porous disk with uniform suction or injection. Fluid dynamics Research, V.23, pp.283-290.

[12] Ibrahim, F.S, Elaiw, A.M and Bakr, A.A : Effect of chemical reaction and radiation absorption on unsteady MHD free convection flow past a semi-infinite vertical 
permeable moving plate with heat source and suction. Comm. In Non-linear Sci. and Num. Simulation, V.13, pp.1056-1066 (2008).

[13] Indudhar Reddy, M, Narasimha Rao, P and Prasada Rao, D.R.V : Effect of chemical reaction and radiation absorption on unsteady MHD double diffusive convective flow of a viscous fluid past a semi-infinite porous plate. J. Pure \& Appl. Phys., V.23, No.2, pp.205-215 (2011).

[14] Krishna,D.V,Prasada rao,D.R.V,Ramachandra Murty,A.S:Hydromagnetic convection flow through a porous medium in a rotating channel., J.Engg. Phy. and Thermo.Phy,V.75(2),pp.281-291(2.

[15] Madhusudhan Reddy, Y, Prasada Rao, D.R.V : Effect of thermo diffusion and chemical reaction on non-darcy convective heat \& mass transfer flow in a vertical channel with radiation. IJMA, V.4, pp.1-13 (2012).

[16] Pai, S.I : Magnetogasdynamics and Plasma dynamics. Springier Verlag, New York (1962).

[17] Prasada Rao, D.R.V, Krishna, D.V and Debnath, L : Combined effect of free and forced convection on MHD flow in a rotating porous channel. Int. J. Math and Math. Sci., V.5, pp.165-182 (1982).

[18] Rao,D.R.V and Krishna,D.V :Hall effects on unsteady hydromagnetic flow., Ind.J.Pure and Appl.Maths,V.12(2),pp.270-276(1981)

[19] Sarojamma, G and Krishna, D.V : Transient Hydromagnetic convection flow in a rotating channel with porous boundaries. Acta Mechanica, V. 39, p.277 (1981).

[20] Sattar, M.A : Free and forced convection boundary layer flow through a porous medium with large suction. Int. J. of Energy Research, V.17, pp.1-17 (1993).

[21] Seth G.S. and Ghosh, S.K : Ind J. Eng. Sci., V.24, No.7, pp.1183-1193 (1986).

[22] Sarkar,D Mukherjee,S:Acta Ciencia Indica.,V.34M,No.2,pp.737-751(2008)

[23] Sato,H:J.Phy.Soc.,Japan,V.16,p.1427(1961)

[24] Seth,G.S,Ansari,S and Ahmad,N : Acta Ciencia Indica ,V.34M, No.4, p.1849 (2008)

[25] Sherman,A and Sutton,G.W:Mhd ,Evanston,Illionis,p.173(1961)

[26] Sivaprasad, R, Prasada Rao, D.R.V and Krishna,D.V: Hall effects on unsteady Mhd free and forced convection flow in a porous rotating

[27] Sreerangavani,K,Rajeswara Rao,U and Prasada Rao,D.R.V:Effect of thermo-diffusion on Mhd convection heat and mass transfer flow of chemically reacting fluid through a porous medium in a rotating system., Presented in $2^{\text {nd }}$ International conference on Applications of Fluid dynamics, July,(2014)

[28] Yamanishi,T:Hall effects on hydromagnetic flow between two parallel plates., Phy. Soc., Japan,Osaka,V.5,p.29(1962). 